\title{
Tumor Laterality Right
}

National Cancer Institute

\section{Source}

National Cancer Institute. Tumor Laterality Right. NCI Thesaurus. Code C160199.

A finding indicating the tumor location is on the right side of the specimen. 